AperTO - Archivio Istituzionale Open Access dell'Università di Torino

Effect of different management protocols for grouping does on aggression and dominance hierarchies

This is a pre print version of the following article:

Original Citation:

Availability:

This version is available http://hdl.handle.net/2318/1740915

since 2020-06-10T10:54:47Z

Published version:

DOI:10.1016/j.applanim.2020.104999

Terms of use:

Open Access

Anyone can freely access the full text of works made available as "Open Access". Works made available under a Creative Commons license can be used according to the terms and conditions of said license. Use of all other works requires consent of the right holder (author or publisher) if not exempted from copyright protection by the applicable law. 


\title{
1 Effect of different management protocols for grouping does on 2 aggression and dominance hierarchies
}

4 Chiara Munari $^{\mathrm{a}, \mathrm{b} *}$, Cecilia Mugnai ${ }^{\mathrm{a}}$, Michèle Braconnier ${ }^{\mathrm{b}}$, Michael Jeffrey Toscano ${ }^{\mathrm{b}}$,

5 Sabine G. Gebhardt-Henrich ${ }^{\mathrm{b}}$

6

${ }^{a}$ Department of Veterinary Sciences, University of Turin, Largo Braccini 2, 10095, Grugliasco, TO, Italy

${ }^{\mathrm{b}}$ Center for Proper Housing of Poultry and Rabbits (ZTHZ), Division of Animal Welfare, VPH Institute, University of Bern, Burgerweg 22, CH-3052 Zollikofen, Switzerland

* corresponding author: chiara.munari@unito.it

\begin{abstract}
The study aimed at evaluating different management protocols of grouping does in regard to aggressive interactions and the establishment of a hierarchy under commercial conditions. Fifty-seven multiparous rabbit does of the Hycole hybrid maternal line were randomly distributed into three different management protocols (MP) for a total of 5 consecutive trials: MP12 with grouping 12 days after parturition, MP18 after 18 days and MP22 after 22 days. Video recordings were made during the first 24 hours after grouping and after 6 and 10 days, and used to score aggressive interactions of the animals with exception of treatment 3 where day 10 was omitted because it was after the weaning of the kits. For MP12 and MP18 the frequency of different categories of aggressive interactions were significantly higher on the day of grouping than afterwards, with a strong decrease on day $6(\mathrm{P}<0.02)$, but almost no change was found between days 6 and 10. This was especially true for biting, with a high frequency of occurrence just after grouping and a subsequent decrease on day 6 under all
\end{abstract}


management schedules $(\mathrm{P}=0.005)$. The change in the number of aggressive interactions between 6 and 10 days after grouping was not significantly different between MPs. However, the time point of 6 days after grouping seemed to be fundamental in reducing the number of aggressive events. Although a longer separation for 18 and 22 days after parturition did not reduce the total number of aggressive interactions, MP18 and MP22 showed more mild aggressive interactions with respect to MP12 on the day of regrouping. Moreover, when comparing MPs concerning the development of hierarchy, hierarchies appeared very stable independently of the length of grouping $(\mathrm{P}<0.01)$.

\section{Keywords}

Rabbit does; Group housing; Management protocol; Aggression and dominance hierarchies; Welfare

\section{Introduction}

Under natural conditions, the European rabbit (Oryctolagus cuniculus) is a social animal and the typical group structure consists of an average of 2-9 females, 2-3 bucks and their litters (Surridge et al., 1999). Wild rabbits establish dominance ranks for access to breeding sites. Low-ranking does are more stressed than high-ranking does as indicated by increased corticosterone challenge values, as well as low reproductive success (Holst et al., 1999). Social behaviours of hybrid breeding does kept for commercial purposes reflect natural behaviours, due to the limited domestication efforts which have not resulted in marked behavioural differences from the wild rabbit (Trocino and Xiccato, 2006). In a Belgian park system where breeding does are group housed during part of the time, physical contact between animals are infrequent and agonistic interactions occur especially after the formation of the group (Buijs and Tuyttens, 2015). However, in commercial farm conditions, agonistic interactions between rabbit does commonly result in mild to severe injuries, reflecting natural behaviour (Rommers et al., 2006). The reason is that in wild rabbits, separate dominance hierarchies are maintained among males and females. The males' hierarchy is quite rigid, with the dominant male, often larger and older than the others, having priority over females for mating and 
the best places to feed and rest. On the contrary, the females' hierarchy is much less rigid and the dominant females are more tolerant towards the other females of the group. However, during the reproductive season, females can become extremely aggressive towards the same does with whom they spent autumn and winter (Cowan and Bell, 1986). The most important resource for does is to find a dry and safe place to build the nest, but these places may not be easily available within commercial systems leading to fighting to establish the right to take over a particular site to give birth (Mc Bride, 2004). Serious aggressive behaviours such as biting usually become less frequent once a stable hierarchy has been established, and strong positive relationships develop between individuals, who remain near each other and rest together (EFSA, 2005). Under commercial farming conditions, when group housing is applied, females are usually regrouped with unfamiliar does, and this causes the establishment of a new hierarchy through fights and aggressive interactions with consequently high rates of injuries and low reproductive efficiency (Szendro and McNitt, 2012; Andrist et al., 2013). On the other hand, conventional single housing of the domestic rabbits in small wire cages, utilized until now in most European countries, often leads to stereotypic behaviours like hair-chewing and biting bars, indicative of anxiety and frustration, as well as skeletal abnormalities (Lehman, 1991; Gunn and Morton, 1995). To solve these welfare problems of rabbit breeding systems, members of the European Parliament's Agriculture Committee voted in favour of a report that set out key improvements for rabbit (growing and does) welfare in January 2017.

Regarding the Swiss farming system, group housing of breeding rabbit does is a requirement for welfare friendly labels though results in many of the problems already introduced necessitating alternatives. In this context, Andrist et al. (2013) found mild to severe lesions due to aggression in up to one third of group-housed animals. The prevalence of injuries was especially high in the management system with does individually separated after parturition for 12 days in order to prevent fighting for nests, two litters in one nest box and pseudo pregnancy due to mounting does. Due to the impossibility of performing basic ethological behaviours in single cages (Gunn and Morton, 1995) 
and a high competition for the nesting sites before parturition and protection of litters from unfamiliar does in colony housing (Mugnai et al., 2009), the industry lacks a management protocol that can ensure a high level of animal welfare. As infanticides are restricted to the first 10 days after parturition when females stay close to their burrows and are more aggressive towards other females in nature (Rödel et al., 2008), does are individually housed during 12 days after parturition and group housed thereafter. In contrast to other farm animals, such as pigs (Hoy et al., 2006) and cattle (Menke et al., 2000), where the effects of regrouping animals have been extensively studied, there are few studies on the development of hierarchy after the grouping of breeding does. Greater understanding of how the dynamics of dominance evolves within the group over time would provide important information to optimize group housing management systems.

The aim of this study was to find the best management protocol for regrouping (different isolation periods to overcome the critical phase after parturition) does, to minimize social conflicts and that can be applied on commercial farms. For this purpose, aggressive behaviour as well as the establishment and stability of dominance hierarchies were observed..

\section{Animals, materials and methods}

\subsection{Animals and housing}

The experiment was carried out in a commercial rabbit farm in Geltwil (Switzerland), using a total of 57 does of the Hycole hybrid maternal line that were not nulliparous, from August 2018 until March 2019. Does were housed in groups of eight animals each, for five consecutive trials. They were reared according to a Swiss animal-friendly label programme, which requires group housing of females and a neparated for each doe (http://www.blw.admin.ch/themen/00006/01715/01718/index.html?lang=de). Each pen was equipped with straw material and furnished with elevated platforms, hiding places, eight 
compartments with nest boxes, drinkers and automatic feeders (Figure 1). Feed (UFA 925, UFA AG, Herzogenbuchsee, Switzerland), water and hay were provided ad libitum.

\subsection{Experimental timing and management protocols}

For each of five trials, all animals were artificially inseminated (AI) on day 10 postpartum $(p p)$ and were housed individually from one day before parturition until day $11 p p$. From this point, does were divided into three different management protocols (MPs) (Figure 2), as follows: group housing from day $12 p p$ (MP12), group housing from day $18 p p$ (MP18), group housing from day 22 $p p$ (MP22).

To avoid the effect of parity order, in trial 1 all does were assigned to each MP semi-randomly in a standardized way to ensure a similar distribution of parities. In consecutive trials, doe groups were assigned to another MP. Does not pregnant as detected by manual palpation were replaced with other animals. At least 2 does were replaced in each MP after each trial to achieve the group size of 8. No group remained stable between trials.

\subsection{Behavioural observations}

Following the methods of Andrist et al. (2012), video sequences were recorded and evaluated (Figure 2) for each management protocol, as follows: MP12 at days 12, 18 and 22 pp, MP18 at days 18, 24 and $28 p p$, MP22 at days 22 and $28 p p$. All groups were observed during the first 24 hours and after 6 and 10 days after regrouping, except for MP22 group in which day 10 was not present because it was after weaning of the kits. All does were individually marked with livestock colour on their backs and had numbered ear tags. 
In accordance with Selzer et al. (2001), active behaviour is more common during dark hours

125 than during light hours, because rabbits are crepuscular animals. Therefore, two time-windows of 4 126 h each between 20:00 and 00:00 and between 04:00 and 08:00, respectively, were analysed for each time point through video recordings with infra-red sensitive cameras. Based on an ethogram by Graf et al. (2011), aggressive interactions were classified as biting (gripping with the teeth), boxing (hitting with the front paws), chasing (aggressive following of another individual for at least three jumps), ripping (two does kicking each other with the hind legs), carousel-fights (rapid chasing around and around in one spot with the rear end of the opponent gripped between their teeth), threatening (quick head movement towards another doe) and attacking (abruptly running towards a group mate). Threatening and attacking were combined as mild aggressions because no body contact and hence no injuries resulted. Likewise, biting and ripping were combined into a single response (severe aggressions) as were chasing and carousel (without biting) (chasing behaviour).

For each agonistic event, the following parameters were recorded: type of aggressive interaction, frequency of occurrence, the animal directing the behaviour (dominant subject), the recipient of the behaviour (submissive animal), and location (classified as own nest, foreign nest, platform, down) (Williamson et al., 2016). Frequencies of all interactions and durations of chasing, ripping and carousel-fights were recorded. Aggressive interactions were considered to have ended when each individual separated and engaged in different behaviours such as self-grooming, feeding etc.

\subsection{Dominance hierarchy analysis}

The analysis of dominance within social animal structure has been a research focus since the beginning of the last century (Schjelderup-Ebbe, 1922) and different methods have been used to determine individual ranks from interactions. Among them, the Elo-rating method (Elo, 1978) tracks rank measures as a consequence of wins and losses in encounters with other individuals: numerically 
greater ratings indicate more successful competitors. Since Neumann et al. (2011) have published an $\mathrm{R}$ function, use of Elo-ratings together with a stability index to model dominance hierarchies has become one of the most useful methods in this field (Mc Donald and Shizuka, 2013).

For assessing the dominance hierarchy of each treatment along the five trials, the index of stability (S) was calculated which ranges between 0 and 1, where 1 refers to stable hierarchies, whereas values closer to 0 indicate more unstable hierarchies (McDonald and Shizuka, 2013).

\subsection{Ethical approval}

This study was approved by the Cantonal Office of Aargau (No. 30611) and met all cantonal and federal regulations of Switzerland.

\subsection{Statistical analysis}

Statistical analysis was conducted using the software package SAS 9.4. Generalized linear models on count data (Poisson distribution) with management protocol, trial, and day as fixed categorical effects were computed using Proc Glimmix [SAS/STAT] Version 13.1 software ${ }^{1}$. Residuals were checked for normality. No videos existed for day 10 after regrouping in MP22 because offspring were weaned before this day which made comparisons with the other protocols not possible. Therefore, two sets of analyses were performed: 1) Comparing days 0, 6, and 10 after regrouping for MP12 and 18 and 2) comparing days 0 and 6 after regrouping for all MPs. P-values were adjusted for multiple comparisons by Tukey-Kramer. The relationship between the number of aggressive interactions and time-of-day was analysed with the regression model including the linear and the square term of time-of-day (Proc Reg). Only time points between 20:00 and 24:00 were included

\footnotetext{
${ }^{1}$ SAS and all other SAS Institute Inc. product or service names are registered trademarks or trademarks of SAS Institute Inc. in the USA and other countries. \& indicates USA registration.
} 
because only 3 aggressive events happened during the second time slot from 4:00 to 8:00. However, the other analyses included all data.

Dominance hierarchy and its stability were calculated with $\mathrm{R}$ (version 3.6.0), using the package EloRating (version 0.46.8, https://cran.r-project.org/web/packages/EloRating/index.html accessed 9-9-2019). Results were assessed as significant when $\mathrm{P}<0.05$.

\section{Results}

Considering MP12 and MP18, the frequency of aggressive interactions decreased noticeably from day 0 after regrouping, but no significant differences were detected between days 6 and 10 (Figure 3). Analysing the number of aggressive acts $(\mathrm{N}=30)$, in the generalized linear model, protocols did not differ (MP: $\mathrm{F}_{1,4}=1.07, \mathrm{P}=0.36$ ), whereas day and a day $\mathrm{x}$ protocol interaction were significant (Day: $\mathrm{F}_{2,8}=13.78, \mathrm{P}=0.003$; Interaction: $\mathrm{F}_{2,8}=4.44, \mathrm{P}=0.05$ ). Regarding the interaction, the variable day was significant only for MP18, but not for MP12 (MP12: $\mathrm{F}_{2,8}=1.28, \mathrm{P}$ $\left.=0.33 ; \mathrm{T} 18 \mathrm{~F}_{2,8}=25.01, \mathrm{P}=0.0004\right)$. When day 10 was deleted in order to include all MPs, they did not differ in the number of aggressive encounters (MP: $F_{2,6}=1.96, P=0.22$; Interaction: $F_{2,6}=4.66$, $P=0.06$ ), however days differed (Day: $F_{1,6}=31.08, P=0.001$ ). The number of aggressive encounters decreased from day 0 to day $6\left(\mathrm{~F}_{1,22}=11.58, \mathrm{P}=0.003, \mathrm{~N}=30\right)$, but no effects of protocol $\left(\mathrm{F}_{2,22}=\right.$ $0.24, \mathrm{P}=0.79, \mathrm{~N}=30)$ and trial $\left(\mathrm{F}_{4,22}=2.54, \mathrm{P}=0.06, \mathrm{~N}=30\right)$ were found when all MPs were considered. Similarly, for MP12 and MP18, biting showed significant differences in respect of the day of observation $\left(\mathrm{F}_{2,22}=6.92, \mathrm{P}=0.005, \mathrm{~N}=30\right)$, but protocol $\left(\mathrm{F}_{1,22}=0.2, \mathrm{P}=0.66, \mathrm{~N}=30\right)$ and trial $\left(\mathrm{F}_{4,22}=1.47, \mathrm{P}=0.25, \mathrm{~N}=30\right)$ did not.

When all MPs were considered, MP18 and MP22 showed more aggressive interactions classified as mild (threats and attacks), than MP12, on the day of regrouping (Table 1) (MP12 vs. 
MP18: $\mathrm{t}_{8}=-3.73, \mathrm{P}_{\mathrm{adj}}=0.045 ; \mathrm{MP12}$ vs. MP22: $\left.\mathrm{t}_{8}=-4.16, \mathrm{P}_{\mathrm{adj}}=0.026\right)$. On day 6 , MPs did not differ (all P-values above 0.39).

Only 3 out of 40 aggressive interactions occurred between 04:00 and 08:00. Disregarding this time slot, the number of aggressive encounters increased with time from 20:00 to midnight $\left(\mathrm{F}_{2,33}=\right.$ $7.11, \mathrm{P}=0.003)$ with a linear $\left(\mathrm{t}_{1}=2.4, \mathrm{P}=0.02\right)$ and an exponential (square) term $\left(\mathrm{t}_{1}=2.5, \mathrm{P}=0.02\right)$ (Figure 4).

\subsection{Dominance hierarchy}

The stability coefficients were very high (mostly above 0.6) and did not differ among MPs $\left(\mathrm{F}_{2,9}=1.10, \mathrm{P}=0.38\right)$. However, trials differed $\left(\mathrm{F}_{4,9}=6.38, \mathrm{P}<0.01\right)$ (Figure 5). In trial 2, the Elorating program did not generate a value of social stability for MP18 and MP22, possibly because the number of interactions was too low.

\section{Discussion}

The aim of the present study was to investigate whether aggressive interactions of breeding does can be reduced by keeping them isolated for more than 12 days after parturition. Being gregarious animals, it would be desirable to keep rabbit does in groups for ethological reasons, but this results in social conflicts with increase of stress and injuries, reducing their welfare and performance (Rommers et al., 2006; Mugnai et al., 2009; Andrist et al., 2012). In addition, there can be aggression towards offspring. As found by Mykytowycz and Dudzinski (1972), does tolerate their own kits, but attack kits from other does. In this respect, Szendrő et al. (2012) recorded a high frequency of bitten and injured litters by competitive does, reducing the chance of survival with lower productive performances and less income for the farmer. However, Albonetti and Farabollini (1994) found a large decrease in terms of aggressive interactions after the establishment of a hierarchy and 
suggested that social interactions between rabbit does are mostly friendly over time, after a first period of fights.

In our study, for MP12 and MP18, the frequency of different categories of aggressive interactions were higher on the day of regrouping, with a dramatic decrease after 6 days. In contrast, almost no change was found between 6 and 10 days suggesting that aggressive interactions ceased after the social rank was established (Albonetti et al., 1990b; Andrist et al., 2013). It has generally been observed that regrouping unfamiliar animals leads to an increase in aggressive behaviours at the moment of group formation (Mykytowycz, 1958; Albonetti et al., 1990a) because a new dominance hierarchy needs to be established (dominance aggression), and also probably to compete for resources and space (territorial aggression) (Mykytowycz, 1958; Graf et al., 2011).

Only the factor number of days after parturition clearly affected the frequency of biting as the most damaging aggressive interaction, with a high frequency of occurrence after regrouping and a subsequent decrease in the course of the six-day observation period. Biting is considered a serious interaction due to the potential for severe injury. Our findings confirm other studies (Mykytowycz, 1958; Lehmann, 1991) showing that, although aggressive chasing and submissive retreat remain common, overt fighting becomes rare after the order of dominance has been established if the group composition remains intact. This suggests that most of the agonistic encounters were caused by dominance aggression because a new hierarchy needed to be established after the animals were grouped, also caused by protection towards the litter (Szendro et al., 2012). In case of territorial aggression agonistic encounters would remain frequent (Mykytowycz, 1958). Moreover, Larsen and Grattan (2012) found that in mice prolactin induces neurogenesis in the female with critical changes in the mood and behavior in the postpartum period. It is well known that prolactin has a complex role in regulating aspects of maternal behaviour (Gonzà et al., 1996), therefore can be probably involved in aggressions, aimed at protecting the kits. When correlating hormonal regulation of maternal behaviour with lactation curve of rabbit does, there seems to be a link between the timing of 
aggressions and milk production, as previously reported by Zomeño et al. (2018). In fact, in our study, MP12 showed the highest frequency in severe aggressive interactions, probably linked to the highest milk output, respect MP18 and MP22 that showed more mild aggressive interactions, probably due to the descent phase of the lactation curve. In rabbits the curve of lactation is asymmetric with a convex ascending and a concave descending period (Lebas, 1968) after the peak of lactation on day 18-19 after parturition (Lebas, 1968).

However, when considering all types of aggression, fewer severe aggressive interactions were present in MP18 than in MP12. In particular, MP18 and MP22 showed more mild aggression without body contact than MP12 on the day of regrouping. Therefore, the level of aggression seemed to be affected by the durations of separation between does, following parturition, probably due to the greater age of kits that were, after a longer isolation, less vulnerable and so does don't need to apply severe aggressive interactions like biting or boxing to protect them.

The change in the frequency of biting between 6 and 10 days after regrouping was not significantly different between protocols, suggesting that a hierarchy was established within a few days and biting mostly stopped.

Aggressive interactions were most frequent in the dark hours following regrouping, in fact hardly any aggressive interactions were recorded during light hours (8.00-20.00) (unpublished data). Since the trials were spread from late summer to spring the relationship between time-of-day and amount of aggressive interactions is difficult to interpret, but the highest frequency was found close to midnight when it was always dark. This can be a problem for farm management because the farmer might not be aware of the aggressive behaviours. 
When animals form social groups, it is possible to determine their order within the dominance 275 hierarchy (Hinde, 1976). According to Elo (1978), individuals with similar Elo-ratings (and thus competitive abilities) may be considered to belong to the same category or class, while dissimilar Elo-ratings are predictive of clear dyadic dominance relationships. To the best of the authors' knowledge, there have not been any studies on the strength or stability of hierarchies in breeding rabbit does before. The observed stabilities were higher than 0.6 in most trials, which indicates stable hierarchies (McDonald \& Shizuka (2013). Moreover, our data displayed a strong effect of trial period which might be due to season since the first trial was carried out in September, the third and fourth in the winter and the fifth in February/March. It appears that the groups outside the natural breeding season, namely in the winter, had lower stabilities. Stability might result from higher aggressive interactions due to a higher level of testosterone, confirmed by Birganti et al. (2003) where an increased testosterone induced agonistic interactions in dominant rabbits. Does had smaller anogenital distances in the winter trials which supports this interpretation (Michèle Braconnier, personal communication).

\section{Conclusions} infanticide and pseudopregnancy. Our results indicate that a longer separation for 18 and 22 days did not reduce the number of total aggressive interactions, but a longer separation than 12 days was important to reduce severe behaviours between does. Additionally, the time point of 6 days after regrouping resulted to be crucial for the reduction of total aggressive interactions, as time frame necessary to establish the hierarchy. Maybe it would be better to give access to the other does gradually e.g. grouping them during the daylight with separation during the night, since hardly any 297 fights occurred during the light hours (unpublished data) being crepuscular animals and so more active at night, thus having a gradual approach to regrouping the animals. 

important suggestion for housing systems in terms of welfare, further investigations (physiological evaluations) should be performed to complement the hierarchy stability measurements, analyzing the possible correlations between milk production (lactation curve), associated hormonal changes and maternal behaviour (kits protection).

\section{Acknowledgements}

This work was supported by the FSVO (Food Safety and Veterinary Office) No. 2.18.04. Yamenah Gomez helped with the Elo program. Markus Schwab provided much needed technical support. Many thanks also go to Felix Näf and his team.

\section{References}

Albonetti, M.E., Dessi-Fulgheri, F., Farabollini, F., 1990a. Organization of behavior in unfamiliar female rabbits. Aggress. Behav. 17, 171-178.

Albonetti, M.E., Dessi-Fulgheri, F., Farabollini, F., 1990b. Intrafemale agonistic interactions in the domestic rabbit (Oryctolagus cuniculus L.). Aggress. Behav. 16, 77-86.

Albonetti, M.E., Farabollini, F., 1994. Social stress by repeated defeat: effects on social behaviour and emotionality. Behav. Brain Res. 62, 187-93.

Andrist, C.A., Bigler, L.M., Würbel, H., Roth, B.A., 2012. Effects of group stability on aggression, stress and injuries in breeding rabbits. Appl. Anim. Behav. Sci. 142 (3-4), 182-188. 
Birganti, F., Della Seta, D., Fontani, G., Lodi, L., Lupo, C., 2003. Behavioural effects of testosterone in relation to social rank in the male rabbit. Aggress. Behav. 29, 269-278.

Buijs, S., Tuyttens, F.A.M., 2015. Evaluating the effect of semi-group housing of rabbit does on their offspring's fearfulness: can we use the open-field test? Appl. Anim. Behav. Sci. 162, 58-66.

Cowan, D.P., Bell, D.J., 1986. Leporid social behaviour and social organization. Mamm. Rev. 16 (34), 169-179.

EFSA (European Food Safety Authority), 2005. "The impact of the current housing and husbandry systems on the health and welfare of farmed domestic rabbits. EFSA journal 267, 1-31.

Elo, A., 1978. The rating of cheesplayers, past and present. New York: Arco.

Gonzà, G., Mariscal, I., Rosenblatt, J.S., 1996. Maternal behavior in rabbits: a historical and multidisciplinary perspective. Adv. Study Behav. 25, 330-360.

Graf, S., Bigler, L., Failing, K., Würbel, H., Buchwalder, T., 2011. Regrouping rabbit does in a familiar or novel pen: Effects on agonistic behaviour, injuries and core body temperature. Appl. Anim. Behav. Sci. 135 (1-2), 121-127.

Gunn, D., Morton, D.B., 1995. Inventory of the behaviour of New Zealand White rabbits in laboratory cages. Appl. Anim. Behav. Sci. 45 (3-4), 277-292.

Hinde, R.A., 1976. Interactions, relationships and social structure. Man. 11, 1-17.

Holst von, D., Hutzelmeyer, H., Kaetzke, P., Kaschei, M., Schönheiter, R., 1999. Social rank, stress, fitness, and life expectancy in wild rabbits. Naturwissenschaften 86, 388-393.

Hoy, S., 2006. Ferkelerzeugung: Sauen gruppieren. Welche Methoden gibt es? DGS Magazin 13, 3639. 
Larsen, C.M., Grattan, D.R., 2012. Prolactin, neurogenesis, and maternal behaviors. Brian, Behavior and Immunity 26, 201-209.

Lebas, F., 1968. Mesure quantitative de la production laitière chez la lapine Ann. Zootech. 17, 169182.

Lehman, M., 1991. Social behaviour in young domestic rabbit under semi-natural conditions. Appl. Anim. Behav. Sci. 32, 269-292.

McBride, A., 2004. Perchè il mio coniglio fa così? Alberto Perdisa Editore, Bologna, Italia, 170 p. McDonald, D.B., Shizuka, D., 2013. Comparative transitive and temporal orderliness in dominance networks. Behav. Ecol. 24, 511-520.

Menke, C., Waiblinger, S., Fölsch, D., 2000. Die Bedeutung von Managementmassnahmen im Laufstall für das Sozialverhalten von Milchkühen. Deutsche tierärztliche Wochenzeitschrift. 107, 253-292.

Mugnai, C., Dal Bosco, A., Castellini, C., 2009. Effect of different rearing systems and pre-kindling handling on behaviour and performance of rabbit does. Appl. Anim. Behav. Sci. 118, 91-100.

Mykytowycz, R., 1958. Social behaviour of an experimental colony of wild rabbits Oryctolagus cuniculus L.; I establishment of the colony. CSIRO Wildl. Res. 3, 7-25.

Mykytowycz, R., Dudzinski, M.L., 1972. Aggressive and Protective Behaviour of Adult Rabbits Oryctolagus cuniculus (L.) towards Juveniles. Behaviour, Vol. 43, pp. 97-120.

Neumann, C., Duboscq, J., Dubuc, C., Ginting, A., Irwan, A. M., Agil, M., Widdig, A., Engelhardt, A., 2011. Assessing dominance hierarchies: Validation and advantages of progressive evaluation with Elo-rating. Anim. Behav. 82, 911-921. 
Rödel, H.G., Starkloff, A., Bautista, A., Friedrich, A.C., Holst, D. von, 2008. Infanticide and Maternal Offspring Defence in European Rabbits under Natural Breeding Conditions. Ethology 114 (1), 2231.

Rommers, J.M., Boiti, C., Jong, I.D., Brecchia, G., 2006. Performance and behaviour of rabbit does in a group-housing system with natural mating or artificial insemination. Reprod. Nutr. Dev. 46 (6), $677-687$.

SAS Institute Inc. 2010. SAS/STAT® 9.22 User's Guide: The GLIMMIX Procedure. Cary, NC: SAS Institute Inc. Page 2698.

Selzer, D., Lange, K., Hoy, S., 2001. Untersuchungen zur Mutter-Kind-Beziehung bei Hauskaninchen unter Berücksichtigung verschiedener Haltungsbedingungen. Proc. 12th Symposium on Housing and Diseases of Rabbits, Furbearing Animals and Pet Animals Celle, 106-114.

Schjelderup-Ebbe, T., 1922. Beiträge zur Sozialpsychologie des Haushuhns. Zeitschrift für Psychologie. 88, 226-252.

Szendrő, Z., McNitt, J.I., 2012. Housing of rabbit does: Group and individual systems: A review. Livest. Sci. 150 (1-3), 1-10.

Surridge, A.K., Bell, D.J., Hewitt, G.M., 1999. From population structure to individual behaviour: Genetic analysis of social structure in the European wild rabbit (Oryctolagus cuniculus). Biol. J. Linn. Soc. Lond. 68 (1-2), 57-71.

Trocino, A., Xiccato, G., 2006. Animal welfare in reared rabbits: A review with emphasis on housing systems. World Rabbit Sci. 14, 77-93.

Williamson, C.M., Lee, W., Curley, J.P., 2016. Temporal dynamics of social hierarchy formation and maintenance in male mice. Anim. Behav. 115, 259-272. 
387 Zomeño, C., Birolo, M., Gratta, F., Zuffellato, A., Xiccato, G., Trocino, A., 2018. Effects of group 388 housing system, pen floor type and lactation management on performance and behaviour in rabbit 389 does. Appl. Anim. Behav. Sci. 203, 55-63. 